\title{
BMJ Open Modified pedicle screw placement at the fracture level for treatment of thoracolumbar burst fractures: a study protocol of a randomised controlled trial
}

\author{
Zhi-Chao Hu, ${ }^{1,2,3}$ Xiao-Bin Li, ${ }^{1,2,3}$ Zhen-Hua Feng, ${ }^{1,2,3}$ Ji-Qi Wang, ${ }^{1,2,3}$ \\ Lan-Fang Gong, ${ }^{4}$ Jiang-Wei Xuan, ${ }^{1,2,3}$ Xin Fu, ${ }^{1,2,3}$ Bing-Jie Jiang, ${ }^{1,2,3}$ Long Wu, ${ }^{1,2,3}$ \\ Wen-Fei $\mathrm{Ni}^{1,2,3}$
}

To cite: Hu Z-C, Li X-B, Feng Z-H, et al. Modified pedicle screw placement at the fracture level for treatment of thoracolumbar burst fractures: a study protocol of a randomised controlled trial. BMJ Open 2019;9:e024110. doi:10.1136/ bmjopen-2018-024110

- Prepublication history for this paper is available online. To view these files, please visit the journal online (http://dx.doi. org/10.1136/bmjopen-2018024110).

Received 9 May 2018 Revised 24 November 2018 Accepted 30 November 2018

Check for updates

(C) Author(s) (or their employer(s)) 2019. Re-use permitted under CC BY-NC. No commercial re-use. See rights and permissions. Published by BMJ.

For numbered affiliations see end of article.

Correspondence to

Dr Wen-Fei Ni;

739961818@qq.com

\section{ABSTRACT}

Introduction The optimal treatment for burst fractures of the thoracolumbar spine is controversial. The addition of screws in the fractured segment has been shown to improve construct stiffness, but can aggravate the trauma to the fractured vertebra. Therefore, optimised placement of two pedicle screws at the fracture level is required for the treatment of thoracolumbar burst fractures. This randomised controlled study is the first to examine the efficacy of diverse orders of pedicle screw placement and will provide recommendations for the treatment of patients with thoracolumbar burst fractures.

Methods and analysis A randomised controlled trial with blinding of patients and the statistician, but not the clinicians and researchers, will be conducted. A total of 70 patients with single $\mathrm{A} 0$ type $\mathrm{A} 3$ or $\mathrm{A} 4$ thoracolumbar fractures who are candidates for application of shortsegment pedicle screws at the fractured vertebral level will be allocated randomly to the distraction-screw and screw-distraction groups at a ratio of 1:1. The primary clinical outcome measures will be the percentage loss of vertebral body height, screw depth in the injured vertebrae and kyphosis (Cobb angle). Secondary clinical outcome measures will be complications, Visual Analogue Scale scores for back and leg pain, neurological function, operation time, intraoperative blood loss, Japanese Orthopaedic Association score and Oswestry Disability Index. These parameters will be evaluated preoperatively, intraoperatively, on postoperative day 3, and at 1, 3, 6, 12 and 24 months postoperatively.

Ethics and dissemination The Institutional Review Board of the Second Affiliated Hospital and Yuying Children's Hospital of Wenzhou Medical University have reviewed and approved this study (batch: LCKY2018-05). The results will be presented in peer-reviewed journals and at an international spine-related meeting after completion of the study.

Trial registration number NCT03384368; Pre-results.

\section{INTRODUCTION}

The diagnosis, classification and treatment of thoracolumbar burst fractures continue to be controversial. ${ }^{1}$ The current goal in the
Strengths and limitations of this study

- This study is the first to investigate the effect of the order of pedicle screw placement at the injury level for thoracolumbar burst fractures.

- This double-blinded, randomised controlled trial is designed to strengthen comparability.

- Most clinical data will be collected prospectively, for at least 24 months postoperatively.

- The size of the study sample limits the power of the observations.

treatment of such fractures is to stabilise the spine, to prevent short-term and long-term deformity and neurological decline while improving the clinical outcome. ${ }^{2}$ Anterior, posterior and combined surgical approaches are used for the treatment of unstable burst fractures, but the optimum approach remains unclear. ${ }^{3}$ Nevertheless, spinal surgeons use the posterior approach more commonly, due to its ease of application, reduction of intraoperative bleeding and low degree of invasiveness. Short-segment pedicle screw fixation (one level above and one level below the fracture level) is used more commonly than long-segment fixation for the treatment of thoracolumbar burst fractures. ${ }^{4-8}$ However, several studies have shown that short-segment pedicle screw fixation is not adequate to achieve and maintain the reduction of thoracolumbar fractures and is associated with an unacceptable rate of failure. ${ }^{9-13}$ Additional transpedicular grafting to support the anterior column has been suggested to prevent failure. $^{4514}$

To achieve stiffer short-segment constructs, some surgeons add pedicle screws at the level of the fractured vertebra. ${ }^{1815}$ Clinically and biomechanically, the placement of two additional screws at the fracture level increases 
the stiffness of the fixation system, and could be useful for anterior column reconstruction. ${ }^{8}$ However, some groups have suggested that pedicle screw placement at the fracture level aggravates the trauma to the fractured vertebra. Here, we optimised the placement of the two pedicle screws at the fracture level. The optimised addition of pedicle screws after distraction of the fractured vertebra may be associated with the relief of trauma at the fracture level. To date, no clinical study has examined the efficacy of this different order of pedicle screw placement at the injury level.

This randomised controlled trial (RCT) was designed to examine whether the new order of pedicle screw placement affects the efficacy of posterior short-segment instrumentation at the fracture level for thoracolumbar burst fractures. The results will provide more reliable evidence and recommendations for the treatment of patients with thoracolumbar burst fractures.

\section{Materials and methods}

All participants will be asked to sign an informed consent form. This trial has been registered at the US National Institutes of Health Clinical Trials Registry: NCT03384368. The protocol conforms to the Standard Protocol Items: Recommendations for Interventional Trials. ${ }^{16}$

\section{Participants}

This study is a parallel group RCT conducted at the Orthopaedic Hospital, Second Affiliated Hospital and Yuying Children's Hospital of Wenzhou Medical University.

\section{Randomisation and blinding}

Participants will be randomly divided into either the screw-distraction (SD) group or the distraction-screw (DS) group based on a permuted blocks randomisation scheme. Using a block size of four in a scheduled computer-generated randomisation programme, the final group assignments will be sealed in opaque envelops. In order to ensure the proper management of the randomisation procedure, the sequence numbers will be marked on the opaque envelope, and the group assignment will be sealed inside. All envelopes will be numbered sequentially. The envelopes will be delivered according to patients' sequence numbers, and the surgeon will be informed of the random numbers and group assignments by either telephone or email. Patients will remain blinded for the allocation until the last questionnaires have been completed. At the final follow-up period, the blind can be released to the patient's request. In addition, the assessor will also be blinded. Figure 1 provides the chart of the trial design.

\section{Inclusion criteria}

1. Age $\geq 18$ years.

2. Single thoracolumbar junction fracture.

3. AO type A3 or A4 fracture.

4. Thoracolumbar Injury Classification and Severity score $>4$ and fracture duration $<2$ weeks.

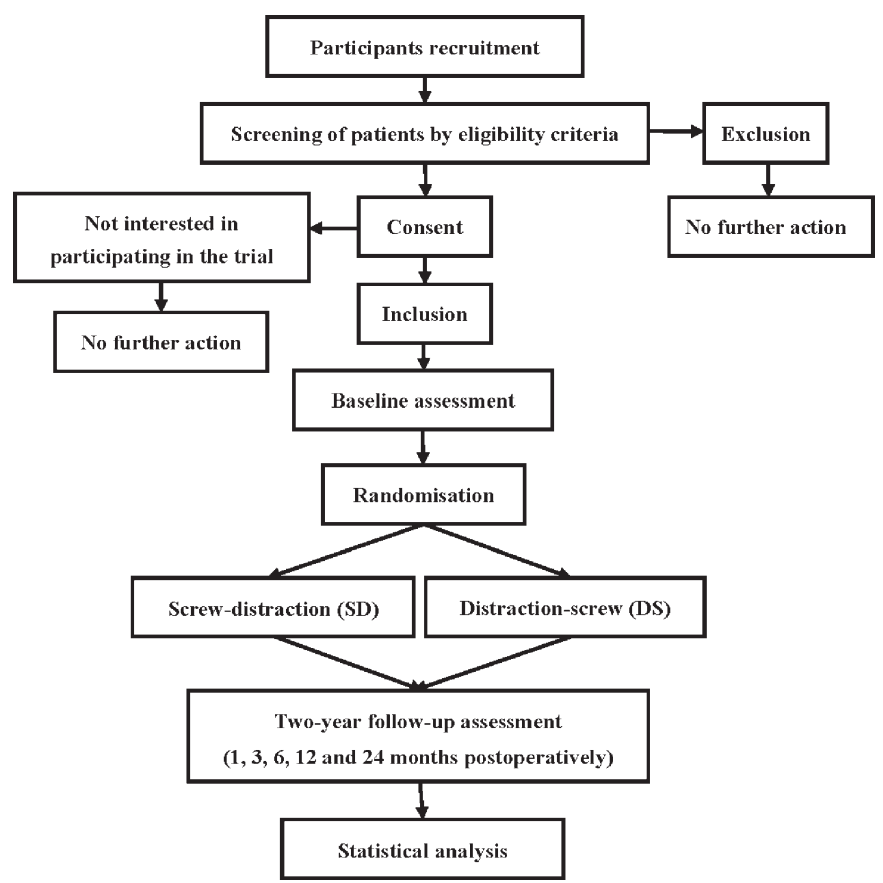

Figure 1 Flow chart showing the steps in participant recruitment, treatment and analysis. SD, six pedicle screws are implanted first, and then distraction is performed; DS, four pedicle screws are implanted first, and then distraction is performed, followed by the placement of two pedicle screws at the fracture level.

5. Application of posterior short-segment pedicle screw instrumentation at the fracture level.

\section{Exclusion criteria}

1. Previous pedicle instrumentation at the same level.

2. Multisegmental thoracolumbar fracture or fracture type other than AO type A3 or A4.

3. Pregnancy.

4. Active infection or previous infection at the surgical site.

5. Planned emigration within 2 years after enrolment.

6. Illness or long-term use of drugs affecting the stability of the spinal environment (eg, metabolic bone disease, spinal tuberculosis).

7. Current use of anticoagulants (eg, warfarin) or postoperative use of heparin for more than 6 months.

\section{Interventions}

SD group

The patient is placed in the prone position with U-shaped pillows under the chest and both ilia so that the abdomen is suspended, and general anaesthesia is applied. A posterior midline skin incision is made at the centre of the fractured vertebra level, identified by X-ray fluoroscopy, to expose the vertebral plate and the articular process layers. According to the technical requirements of pedicle screw placement, four pedicle screws are inserted into the vertebrae one level above and one level below the fractured vertebra. Then, two pedicle screws are inserted into the injured vertebral body. The length and diameter of the 


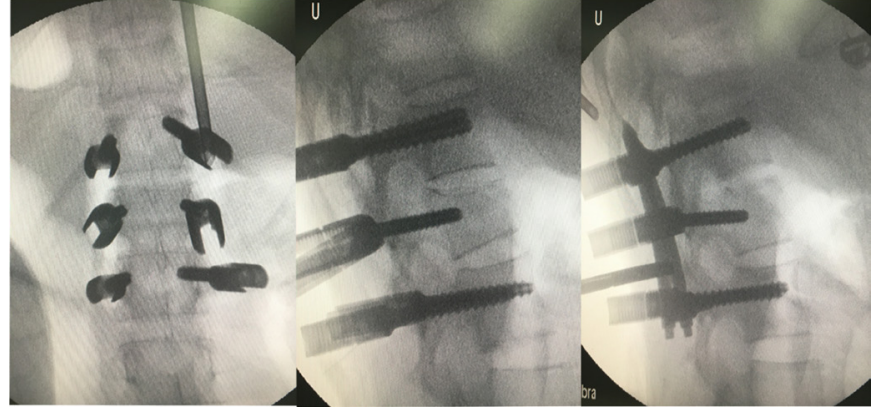

Figure 2 An example of SD order surgery using X-ray fluoroscopy for thoracolumbar burst fractures. All photographs were collected from the Second Affiliated Hospital and Yuying Children's Hospital of Wenzhou Medical University. SD, six pedicle screws are implanted first, and then distraction is performed. SD, screw distraction.

pedicle screws are chosen according to the characteristics of the diverse fixed segments. After the insertion of all screws and application of appropriate curvature to the rod, the assembly is performed. Distraction is applied to correct the deformity and to achieve indirect decompression, followed by tightening of the construct. No patient will undergo laminectomy or fusion. Thorough homoeostasis is achieved and the wound is closed in layers over the drain. Clean dressing is applied (figure 2).

\section{DS group}

The patient is placed in the prone position with U-shaped pillows under the chest and both ilia so that the abdomen is suspended, and general anaesthesia is applied. A posterior midline skin incision is made at the centre of the fractured vertebra level, identified by X-ray fluoroscopy, to expose the vertebral plate and the articular process layers. In the DS group, four pedicle screws are first inserted into the vertebrae one level above and one level below the fractured vertebra. After connecting the rods and screws, distraction force is applied using spreader forceps to restore lordosis and body height. Then, we remove the titanium rods on one side and insert the pedicle screw at the fracture level. Next, we replace and fix the rod. The same operation is performed on the contralateral side. Thus, six pedicle screws are placed in both groups. No patient will undergo laminectomy or fusion. Thorough homoeostasis is achieved and the wound is closed in layers over the drain. Clean dressing is applied (figure 3).

\section{Outcome measurements}

\section{Primary outcomes}

1. The percentage loss of vertebral body height will be calculated using the ratio of the anterior height of the injured vertebra to the mean anterior height of the two adjacent intact vertebrae. It will be evaluated on lateral radiographs or CT sagittal sections preoperatively, intraoperatively, on postoperative day 3 , and at 1, 3, 6, 12 and 24 months postoperatively.

2. The screw depth in the injured vertebra will be calculated using the ratio of the vertical distance between

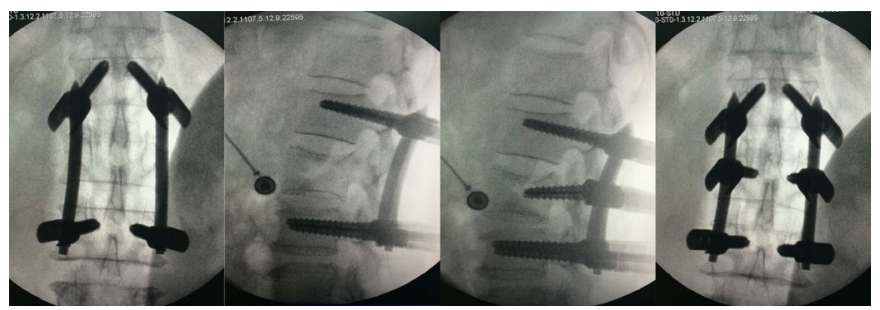

Figure 3 An example of DS order surgery using X-ray fluoroscopy for thoracolumbar burst fractures. All photographs were collected from the Second Affiliated Hospital and Yuying Children's Hospital of Wenzhou Medical University. DS, four pedicle screws are implanted first, and then distraction is performed, followed by the placement of two pedicle screws at the fracture level. DS, distraction screw.

the tip of the screw and the posterior wall of the vertebral body to the distance between the anterior and posterior walls of the vertebral body. It will be evaluated intraoperatively by X-ray fluorescence.

3. Kyphosis (Cobb) angle. Based on a previous report, ${ }^{17}$ local kyphosis will be assessed by measuring the Cobb angle between the superior and inferior end plates of the fractured vertebra. It will be evaluated by X-ray fluorescence preoperatively, intraoperatively, on postoperative day 3 , and at $1,3,6,12$ and 24 months postoperatively.

\section{Secondary outcomes}

1. The degree of pain in the back and lower limbs during follow-up will be assessed using a Visual Analogue Scale (VAS). VAS scores for back pain and leg pain will be recorded preoperatively, on postoperative day 3 , and at 1, 3, 6, 12 and 24 months postoperatively.

2. Oswestry Disability Index (ODI) scores will be recorded preoperatively, on postoperative day 3 , and at 1,3 , 6, 12 and 24 months postoperatively.

3. Japanese Orthopaedic Association (JOA) scores will be recorded preoperatively, on postoperative day 3 , and at 1, 3, 6, 12 and 24 months postoperatively. Functional improvement will be expressed by the rate of recovery of the JOA score.

4. Operative time and intraoperative blood loss will be recorded immediately after surgery.

5. Pedicle fracture, intraoperative pars fracture, postoperative infection, deep venous thrombosis, nerve injury and any other direct or indirect surgical complication will be recorded.

6. The American Spinal Injury Association (ASIA) impairment scale will be evaluated preoperatively, on postoperative day 3, and at 1, 3, 6, 12 and 24 months postoperatively.

Table 1 presents the data collection times.

\section{Baseline demographics}

Sex, age, body mass index, smoking habit, diagnosis, level and occurrence of diabetes will be recorded. 
Table 1 Time of data collection

\begin{tabular}{|c|c|c|c|c|c|c|c|c|}
\hline Measures & $\begin{array}{l}\text { Baseline per } \\
\text { operation }\end{array}$ & $\begin{array}{l}\text { Operation } \\
\text { duration }\end{array}$ & $\begin{array}{l}\text { Follow-up } \\
\text { day } 3\end{array}$ & 1 month & 3 months & 6 months & 12 months & 24 months \\
\hline $\begin{array}{l}\text { Screening for inclusion/ } \\
\text { exclusion criteria }\end{array}$ & $\sqrt{ }$ & & & & & & & \\
\hline Informed consent & $\sqrt{ }$ & & & & & & & \\
\hline Assignment to two groups & $\sqrt{ }$ & & & & & & & \\
\hline Baseline demographics & $\sqrt{ }$ & & & & & & & \\
\hline Operative time & & $\sqrt{ }$ & & & & & & \\
\hline Blood loss & & $\sqrt{ }$ & & & & & & \\
\hline Complications & & $\sqrt{ }$ & $\sqrt{ }$ & $\sqrt{ }$ & $\sqrt{ }$ & $\sqrt{ }$ & $\sqrt{ }$ & $\sqrt{ }$ \\
\hline VAS of back pain & $\sqrt{ }$ & & $\sqrt{ }$ & $\sqrt{ }$ & $\sqrt{ }$ & $\sqrt{ }$ & $\sqrt{ }$ & $\sqrt{ }$ \\
\hline VAS of leg pain & $\sqrt{ }$ & & $\sqrt{ }$ & $\sqrt{ }$ & $\sqrt{ }$ & $\sqrt{ }$ & $\sqrt{ }$ & $\sqrt{ }$ \\
\hline $\mathrm{X}$-ray/CT & $\sqrt{ }$ & $\sqrt{ }$ & $\sqrt{ }$ & $\sqrt{ }$ & $\sqrt{ }$ & $\sqrt{ }$ & $\sqrt{ }$ & $\sqrt{ }$ \\
\hline ODI & $\sqrt{ }$ & & $\sqrt{ }$ & $\sqrt{ }$ & $\sqrt{ }$ & $\sqrt{ }$ & $\sqrt{ }$ & $\sqrt{ }$ \\
\hline JOA & $\sqrt{ }$ & & $\sqrt{ }$ & $\sqrt{ }$ & $\sqrt{ }$ & $\sqrt{ }$ & $\sqrt{ }$ & $\sqrt{ }$ \\
\hline ASIA & $\sqrt{ }$ & & $\sqrt{ }$ & $\sqrt{ }$ & $\sqrt{ }$ & $\sqrt{ }$ & $\sqrt{ }$ & $\sqrt{ }$ \\
\hline
\end{tabular}

ASIA, American Spinal Injury Association; JOA, Japanese Orthopaedic Association; ODI, Oswestry Disability Index; VAS, Visual Analogue Scale.

\section{Follow-up}

Follow-up will be conducted preoperatively, intraoperatively, on postoperative day 3 , and at $1,3,6,12$ and 24 months postoperatively.

\section{Monitoring}

All investigators who have completed good clinical practice training will independently collect the data and assess the clinical outcomes of the treatments. Safety and data monitoring will be performed periodically during the study. Only the principal investigator (W-FN) will have access to the final trial data set. All paper and electronic versions of the case reports will be stored for 10 years in the secure research archives at the Second Affiliated Hospital and Yuying Children's Hospital of Wenzhou Medical University with restricted access.

\section{Sample size calculation}

As no previous similar trial has been conducted using our RCT design, we performed a power analysis to determine the sample size required to show safety with a type I error probability of $5 \%$ and an $80 \%$ probability of avoiding a type II error. In related studies, ${ }^{19} 19$ the mean of the control group was 0.891 and the mean of the intervention group was 0.914 ( $\sigma=3$ was adopted). We carried out two independent samples t-tests using the Power Analysis and Sample Size software and obtained a result of 28. We propose to enrol 56 participants (28 randomised to each arm) and suggest recruitment of an effective sample size of at least 70 participants to allow for a $20 \%$ drop-out rate.

\section{Statistical analysis}

Data from the trial will be analysed using SPSS V.18.0 software. Differences between baseline and follow-up time points, as well as differences between the SD and DS groups in operative time, intraoperative blood loss, percentage loss of vertebral body height, screw depth in the injured vertebrae, kyphosis (Cobb angle), VAS scores, ODI score, JOA score and ASIA score will be analysed by independent samples t-tests with a type I error probability of $5 \%$. The occurrence of complications will be compared using the $\chi^{2}$ test. The compression ratio of the anterior border (vertebral body height), Cobb angle, VAS scores, ODI score, JOA score and ASIA score, evaluated preoperatively, intraoperatively, on postoperative day 3 , and at 1 , $3,6,12$ and 24 months postoperatively, will be analysed by repeated-measures analysis of variance.

\section{Patient and public involvement}

Neither patients nor the public were involved in the development of the research questions, selection of outcome measures, study design or study conduct.

\section{Discussion}

The optimal surgical management of thoracolumbar burst fractures remains controversial, and no evidencebased guideline for the most suitable surgical approach or instrumentation technique has been developed. ${ }^{15}$ 20-23 Posterior transpedicular short-segment instrumentation is the most frequently applied surgical treatment for such fractures because it is associated with low rates of morbidity and comorbidity. ${ }^{5112425}$

The demand for thoracolumbar burst fracture surgery has increased markedly over the last 10 years and is expected to increase further in the near future. This paper describes the rationale and protocol for an RCT to be conducted in China to investigate the efficacy of 
different orders of pedicle screw placement at the level of thoracolumbar burst fractures. The trial is designed to include a control SD group for the comparison of clinical outcomes with those in the DS group. In the SD group, two pedicle screws are placed at the fracture level before distraction. The placement of these screws directly into the vertebra without reduction may render the vertebra vulnerable, affecting the subsequent reduction of the fracture and potentially resulting in fracture displacement. Conversely, in the DS group, pedicle screws are placed after distraction has been performed. Reduction of the vertebral body before the application of pedicle screws will result in less damage to the fractured vertebra. We hypothesise that DS will be superior to SD, reducing the compression ratio of the anterior border (vertebral body height) and improving other clinical outcomes. If this hypothesis is found to be correct, a less invasive order of pedicle screw placement at the injury level will be developed.

This study is the first RCT to compare SD with DS surgery. An RCT has superiority in controlling for all possible variables due to random sequence generation, in which confounding factors and bias may be more problematic. High-quality RCTs are often deemed to be the gold standard for investigation of the effectiveness of an intervention. However, follow-up bias remains the greatest limitation of an RCT.

Randomised trials that compare surgical with non-surgical treatments have several features that are distinct from drug trials and can lead to serious limitations. Moreover, compared with drug trials, surgery has many irreversible features.

If our hypothesis is confirmed, our findings will be important for the scheduling and development of treatment options for thoracolumbar burst fractures. We anticipate that the results will provide more reliable evidence and more reasonable choices for surgeons for the treatment of patients with thoracolumbar burst fractures.

\section{ETHICS AND DISSEMINATION}

The procedure will be performed following the principles described in the Declaration of Helsinki. All of the participants will sign their informed consent. This trial has been registered at the US National Institutes of Health Clinical Trials Registry: NCT03384368. The results will be presented in peer-reviewed journals and an international spine-related meeting after completion of the study.

\footnotetext{
Author affiliations

${ }^{1}$ Department of Orthopedics, The Second Affiliated Hospital and Yuying Children's Hospital of Wenzhou Medical University, Wenzhou, China

${ }^{2}$ Department of the Second School of Medicine, Wenzhou Medical University, Wenzhou, China

${ }^{3}$ Department of Bone Research Institute, The Key Orthopaedic Laboratory of Zhejiang Province, Wenzhou, China

${ }^{4}$ Department of Respiratory Medicine, The First Affiliated Hospital of Wenzhou Medical University, The First Medical School of the Wenzhou Medical University, Wenzhou, China
}

Contributors Z-CH and W-FN helped to conceive and design the trial and wrote the manuscript. J-QW and J-WX contributed to revise the manuscript. XF and B-JJ were responsible for the recruitment and conduction of patients. X-BL and L-FG were the principal investigators of this trial. LW planned the statistical analysis. Z-HF would supervise the trial. All authors read and approved the final manuscript.

Funding This work is supported by Natural Science Foundation of Zhejiang Province (grant no. LY17H060009).

Disclaimer The funders had no role in the design, execution or writing of the study. Competing interests None declared.

Patient consent for publication Not required.

Ethics approval This study conforms to the Declaration of Helsinki and is approved by the Institutional Ethics Review Board of the Second Affiliated Hospital and Yuying Children's Hospital of Wenzhou Medical University ((batch: LCKY2018-05).

Provenance and peer review Not commissioned; externally peer reviewed.

Open access This is an open access article distributed in accordance with the Creative Commons Attribution Non Commercial (CC BY-NC 4.0) license, which permits others to distribute, remix, adapt, build upon this work non-commercially, and license their derivative works on different terms, provided the original work is properly cited, appropriate credit is given, any changes made indicated, and the use is non-commercial. See: http://creativecommons.org/licenses/by-nc/4.0/.

\section{REFERENCES}

1. Kose KC, Inanmaz ME, Isik C, et al. Short segment pedicle screw instrumentation with an index level screw and cantilevered hyperlordotic reduction in the treatment of type-A fractures of the thoracolumbar spine. Bone Joint J 2014;96-B:541-7.

2. Parker JW, Lane JR, Karaikovic EE, et al. Successful short-segment instrumentation and fusion for thoracolumbar spine fractures: a consecutive 41/2-year series. Spine 2000;25:1157-70.

3. Modi HN, Chung KJ, Seo IW, et al. Two levels above and one level below pedicle screw fixation for the treatment of unstable thoracolumbar fracture with partial or intact neurology. J Orthop Surg Res 2009;4:28.

4. Alanay A, Acaroglu E, Yazici M, et al. Short-segment pedicle instrumentation of thoracolumbar burst fractures: does transpedicular intracorporeal grafting prevent early failure? Spine 2001;26:213-7.

5. Cho DY, Lee WY, Sheu PC. Treatment of thoracolumbar burst fractures with polymethyl methacrylate vertebroplasty and shortsegment pedicle screw fixation. Neurosurgery 2003;53:1354-61.

6. Louis CA, Gauthier VY, Louis RP. Posterior approach with louis plates for fractures of the thoracolumbar and lumbar spine with and without neurologic deficits. Spine 1998;23:2030-9.

7. Tezeren G, Kuru I. Posterior fixation of thoracolumbar burst fracture: short-segment pedicle fixation versus long-segment instrumentation. J Spinal Disord Tech 2005;18:485-8.

8. Mahar A, Kim C, Wedemeyer M, et al. Short-segment fixation of lumbar burst fractures using pedicle fixation at the level of the fracture. Spine 2007;32:1503-7.

9. Gurwitz GS, Dawson JM, McNamara MJ, et al. Biomechanical analysis of three surgical approaches for lumbar burst fractures using short-segment instrumentation. Spine 1993;18:977-82.

10. Kramer DL, Rodgers WB, Mansfield FL. Transpedicular instrumentation and short-segment fusion of thoracolumbar fractures: a prospective study using a single instrumentation system. J Orthop Trauma 1995;9:499-506.

11. McCormack T, Karaikovic E, Gaines RW. The load sharing classification of spine fractures. Spine 1994;19:1741-4.

12. Dubousset J. Early failure of short-segment pedicle instrumentation for thoracolumbar fractures. A preliminary report. $J$ Bone Joint Surg Am 1995;77:648-9.

13. Sasso RC, Cotler HB. Posterior instrumentation and fusion for unstable fractures and fracture-dislocations of the thoracic and lumbar spine. A comparative study of three fixation devices in 70 patients. Spine 1993;18:450-60.

14. Oner FC, Verlaan JJ, Verbout AJ, et al. Cement augmentation techniques in traumatic thoracolumbar spine fractures. Spine 2006;31:S89-95.

15. Guven O, Kocaoglu B, Bezer M, et al. The use of screw at the fracture level in the treatment of thoracolumbar burst fractures. $J$ Spinal Disord Tech 2009;22:417-21. 
16. Chan AW, Tetzlaff JM, Gøtzsche PC, et al. SPIRIT 2013 explanation and elaboration: guidance for protocols of clinical trials. BMJ 2013;346:e7586.

17. Dobran M, Nasi D, Brunozzi D, et al. Treatment of unstable thoracolumbar junction fractures: short-segment pedicle fixation with inclusion of the fracture level versus long-segment instrumentation. Acta Neurochir 2016;158:1883-9.

18. Sun C, Liu X, Tian J, et al. Comparison of unilateral versus bilateral pedicle screw fixation at the level of fracture using posterior short-segment pedicle instrumentation in the treatment of severe thoracolumbar burst fractures. Int $J$ Surg 2017;41:50-5.

19. Huang W, Luo T. Efficacy analysis of pedicle screw internal fixation of fractured vertebrae in the treatment of thoracolumbar fractures. Exp Ther Med 2013;5:678-82.

20. Altay M, Ozkurt B, Aktekin CN, et al. Treatment of unstable thoracolumbar junction burst fractures with short- or long- segment posterior fixation in magerl type a fractures. Eur Spine $J$ 2007:16:1145-55.

21. Cheng LM, Wang JJ, Zeng ZL, et al. Pedicle screw fixation for traumatic fractures of the thoracic and lumbar spine. Cochrane Database Syst Rev 2013;26:Cd009073.

22. Farrokhi MR, Razmkon A, Maghami Z, et al. Inclusion of the fracture level in short segment fixation of thoracolumbar fractures. Eur Spine $J$ 2010;19:1651-6.

23. Hu R, Mustard CA, Burns C. Epidemiology of incident spinal fracture in a complete population. Spine 1996;21:492-9.

24. An HS, Simpson JM, Ebraheim NA, et al. Low lumbar burst fractures: comparison between conservative and surgical treatments. Orthopedics 1992;15:367-73.

25. Dick W, Kluger P, Magerl F, et al. A new device for internal fixation of thoracolumbar and lumbar spine fractures: the 'fixateur interne'. Paraplegia 1985;23:225-32. 